\title{
Lithographically fabricated nanopore-based electrodes for electrochemistry
}

\author{
Serge G. Lemay, Dennis M. van den Broek, Arnold J. Storm, Diego Krapf, Ralph M. M. Smeets, \\ Hendrik A. Heering and Cees Dekker \\ Kavli Institute of Nanoscience, Delft University of Technology \\ Lorentzweg 1, 2628 CJ Delft, The Netherlands
}

\section{Supporting Information}

\section{Chemicals}

The measurements were carried out in aqueous solution with $1 \mathrm{mM}$ ferrocenylmethyl-trimethylammonium ions $\left(\right.$ FcTMA $^{+}$) as redox-active species, $1 \mathrm{mM} \mathrm{PF}_{6}{ }^{-}$as the counter-ion, and $1 \mathrm{M} \mathrm{NH}_{4} \mathrm{NO}_{3}$ as supporting electrolyte. The ferrocene derivative was purchased in the form of ferrocenylmethyltrimethylammonium iodide crystals from Lancaster (Eastgate, England). Because the iodide counter-ion reacts with gold and interferes with the ferrocene electrochemistry, it was replaced with $\mathrm{PF}_{6}{ }^{-}$using the following procedure. 25 mg of FcTMA iodide was dissolved in $2 \mathrm{ml}$ Milli-Q water (yielding a $32 \mathrm{mM}$ solution), and the result was mixed with $100 \mu \mathrm{l} 5 \mathrm{M}$ ammonium hexafluorophosphate, $\mathrm{NH}_{4} \mathrm{PF}_{6}$ (Aldrich, USA). The less soluble $\mathrm{PF}_{6}{ }^{-}$ salt of FcTMA ${ }^{+}$precipitates in the presence of excess $\mathrm{NH}_{4} \mathrm{PF}_{6}$, after which it can be separated by centrifugation. Residual iodide was removed by washing the pellet (suspension followed by centrifugation), first with $2 \mathrm{ml} 0.25 \mathrm{M} \mathrm{NH}_{4} \mathrm{PF}_{6}$, then with $2 \mathrm{ml}$ cold Milli-Q water to also remove residual $\mathrm{NH}_{4} \mathrm{PF}_{6}$. The resulting FCTMA $\mathrm{PF}_{6}$ pellet was nearly free of iodide (less than $0.1 \%$ ). 


\section{Electrochemical Measurements}

As illustrated in Figure S1, a small ( 200 $\mu \mathrm{l})$ liquid cell was formed above the electrodes by pressing a cylindrical chamber made of silicone elastometer (Sylgard 184, Dow Corning, USA) against the surface of the silicon wafer. The cell had a circular opening at the top (4 mm diameter) for positioning a reference electrode and a smaller (100-300 $\mu \mathrm{m}$ diameter) circular opening at the bottom. The latter reduced the contact area between the liquid and the silicon oxide surface and thus the capacitance between the solution and the underlying silicon. A stereo microscope (Olympus) and a three-axis manipulator (Newport) were used to align the bottom cell opening with the electrode. Two teflon tubes connected to holes in the walls of the liquid cell allowed easy substitution of the working solution. All sampled-current voltammetry data were recorded in a two-electrode configuration using a Keithley 6430 sub-femtoampere sourcemeter with remote preamplifier. The instrument was controlled via the built-in GPIB interface using in-house Labview software. All electrochemical potentials were measured with respect to a Ag/AgCl reference electrode (model RE-6, BAS, USA). The liquid cell and preamplifier were placed in a home-built Faraday cage.

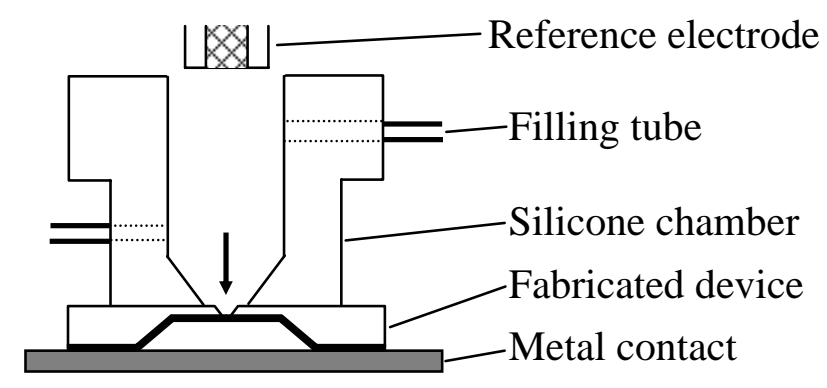

Figure S1. Schematic diagram of the measurement apparatus. The vertical arrow indicates the point where liquid makes contact with the nanoelectrode (the actual electrode is not drawn to scale).

\section{Numerical calculations}

The numerical calculations were performed using Femlab (Comsol AB, Stockholm). Steady-state solutions to the diffusion equation were obtained over a finite three-dimensional volume with a hemispherical outer boundary. This boundary was sufficiently far from the electrode that the diffusion profile was to a very good approximation spherically symmetric at the boundary. The numerical solution inside the finite volume could thus be matched at the outer boundary to the analytical solution for spherical diffusion in an infinite volume. The solutions thus correspond to the steady-state current. The simulations used $F D c_{0}=50 \mathrm{pA} / \mu \mathrm{m}$, as discussed in the main text. 


\section{Interpretation of the calculated diffusion profiles}

The reason why the limiting current is not simply proportional to the lateral dimension $l$ of the electrode is easily understood. For large electrodes $(l>>h)$, the thickness of the membrane is negligible. The diffusion profile (shown in Figure S2a) then effectively corresponds to spherical diffusion with a correction due to the fact that the electrode is not hemispherical. In this regime the limiting current is simply proportional to $l$, the finite-element calculations giving $i_{\text {lim }}=2.1\left(F D c_{0}\right) l$. When $l$ becomes comparable to $h$, however, the concentration gradient in the membrane region becomes large enough that a significant fraction of the total change in concentration between the bulk and the electrode surface occurs across the thickness of the membrane (Figure S2b). $i_{\text {lim }}$ is therefore suppressed. This suppression becomes increasingly pronounced as the ratio $l / h$ decreases, until the regime $l / h<<1$ is reached. In the latter case most of the concentration drop between bulk and the electrode surface occurs in the inverted pyramid hole in the membrane. Diffusion becomes effectively spherical again, albeit with a reduced solid angle corresponding to that of the inverted pyramid. In this regime, we thus again have $i_{\lim } \propto l$, but with a reduced proportionality constant $i_{\lim }=1.1\left(F D c_{0}\right) l$. This regime is not fully developed in our devices because an additional suppression of the current occurs when $l$ becomes comparable to the last remaining length scale in the problem, $h^{\prime}$. The narrow channel near the electrode is then the dominant barrier to diffusion (Figure S2c). There is once again a gradual suppression of the current with decreasing $l$, with the difference that $i_{\lim }$ does not become proportional to $l$ again in the limit $l<<h^{\prime}$. This is because diffusion in the $h^{\prime}$ channel is linear instead of spherical, yielding $i_{\text {lim }} \propto l^{2}$ in this regime. These observations are summarized in Figure S2d.
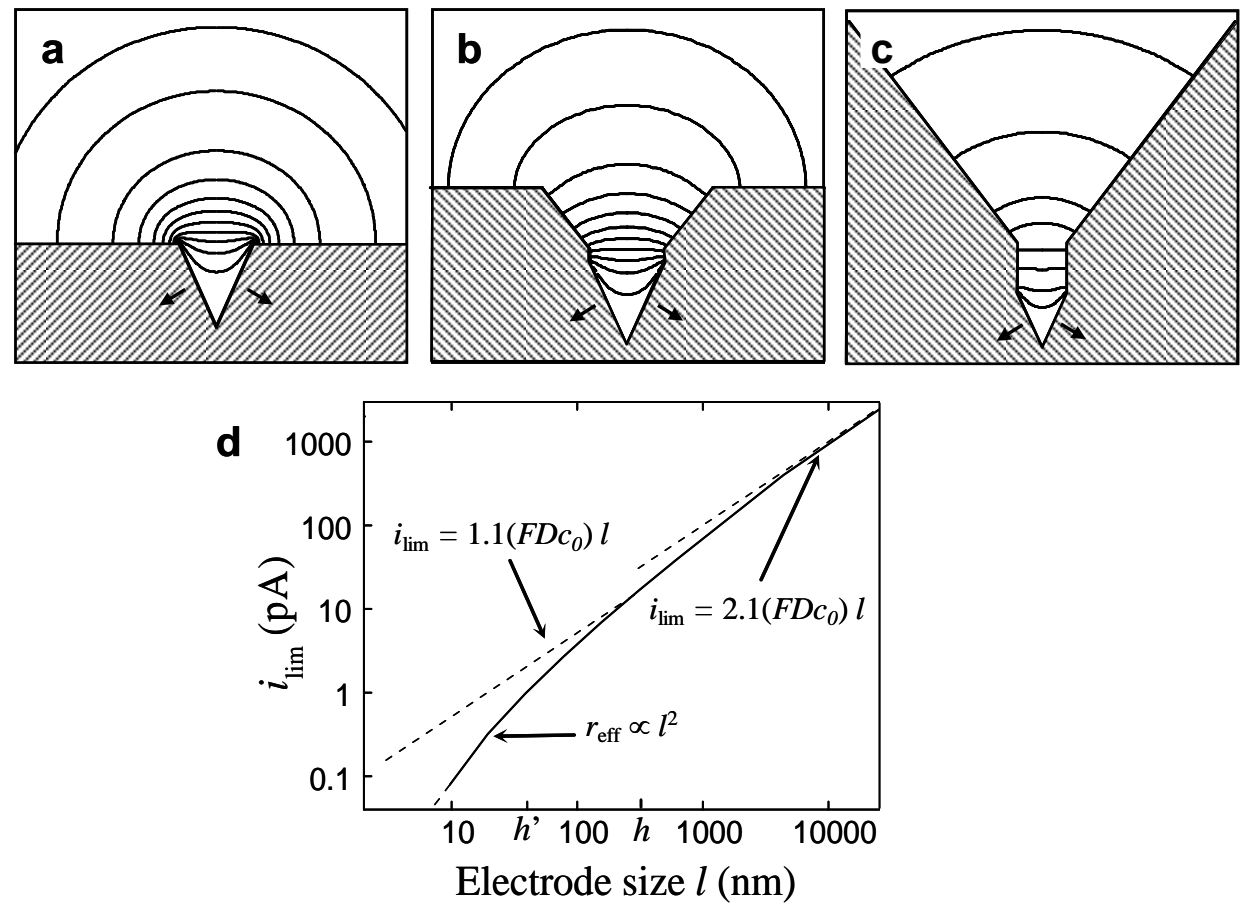

Figure S2. a Contour plot of $c(\boldsymbol{r}) / c_{0}$. The data are plotted on the same two-dimensional plane as in a, and correspond to the cases $h / l=h^{\prime} / l=0$. The contour lines correspond to $c(\boldsymbol{r}) / c_{0}=0.02,0.1,0.2$, $0.3,0.4,0.5,0.6,0.7,0.8,0.9$ and 0.95 . b Same as a for the case $l / h=1, h^{\prime} / h=0.13$. c Same as a for the case $l / h=0.125, h^{\prime} / h=0.13$. The contour lines correspond to $c(\boldsymbol{r}) / c_{0}=0.02,0.1,0.3,0.5,0.7$, 0.8, 0.9 and 0.95 . $\mathbf{d}$ Limiting current $i_{\text {lim }}$ versus electrode size $l$ showing the three main regimes; see above for details. 